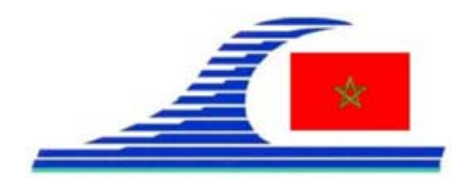

\title{
Vertical porous membrane barrier for coastal structure near a wall
}

\author{
Joydip BHATTACHARJEE ${ }^{1}$, Carlos GUEDES SOARES ${ }^{1}$
}

\author{
1. Centre for Marine Technology and Engineering (CENTEC), \\ Instituto Superior Técnico, Technical University of Lisbon, \\ Av. Rovisco Pais, 1049-001, Lisboa, Portugal. \\ guedess@mar.ist.utl.pt
}

\begin{abstract}
:
The performance of a vertical porous membrane barrier as a breakwater to reduce the wave load on a floating structure near a wall is investigated using linearized water wave theory. The physical problem is modeled in the two dimensional Cartesian coordinate system. A rigid rectangular box is floating in water of finite depth near a rigid vertical wall. The porous membrane breakwater touches the bottom and the tip of the membrane is submerged. Both ends of the membrane are assumed to be kept fixed. The method of eigenfunction expansion and the corresponding orthogonal mode-coupling relations are used to obtain an analytic solution to the boundary value problem. The effect of porosity and tension of the membrane on the wave transmission, wave length and wave amplitude is analyzed.
\end{abstract}

\section{Keywords:}

Vertical porous membrane - Floating structure - Vertical wall - Linear wave theory Eigenfunction expansion - Breakwater

\section{Introduction}

In the past two decades, there has been a growing interest in using flexible membrane as a portable and sacrificial breakwater in various coastal situations. Membrane barriers are lightweight, easily deployable and removable and hence environmental friendly. They can be air dropped via an inflatable buoy and self erected. In coastal engineering, partial bottom mounted barrier is often preferred as it not only reduces the wave amplitude at the resonance, but also allows the navigation of vessels and controls the coastal erosion by restricting the sediment transport (YIP et al., 2002).

The performance of vertical tensioned single and dual membranes spanning the entire water depth were studied by KIM \& KEE (1996), KEE \& KIM (1997), LO (2000) and LEE \& LO (2002) and the literature cited therein.

In addition to the flexible nature of the breakwater, porous structures are often suggested for breakwater systems as they are more efficient in dissipating the incident wave energy and thus reducing the wave load on the barrier (CHWANG \& CHAN, 1998). The porosity of the barrier enables the passage of the underwater stream. 
La connaissance de la Mer :

un vecteur du développement durable en Méditerranée

Different aspects of flexible, porous breakwater systems are analyzed by LEE \& CHWANG (2000), SAHOO et al. (2000), YIP et al. (2002), SURESH KUMAR et al. (2007) and the literature cited therein.

In the present study, the performance of a vertical porous membrane as a breakwater in order to reduce the wave load on a floating structure near a wall is investigated. The effect of the porosity constant and the tension along the membrane on the wave transmission, wave length and wave amplitude is analyzed. In addition, the horizontal deflection of the membrane is also evaluated.

\section{Mathematical formulation}

In the two-dimensional Cartesian coordinate system, the problem is described in a schematic diagram as shown in Fig.1. The body is considered fixed by attachments to the wall, which do not affect the flow filed.

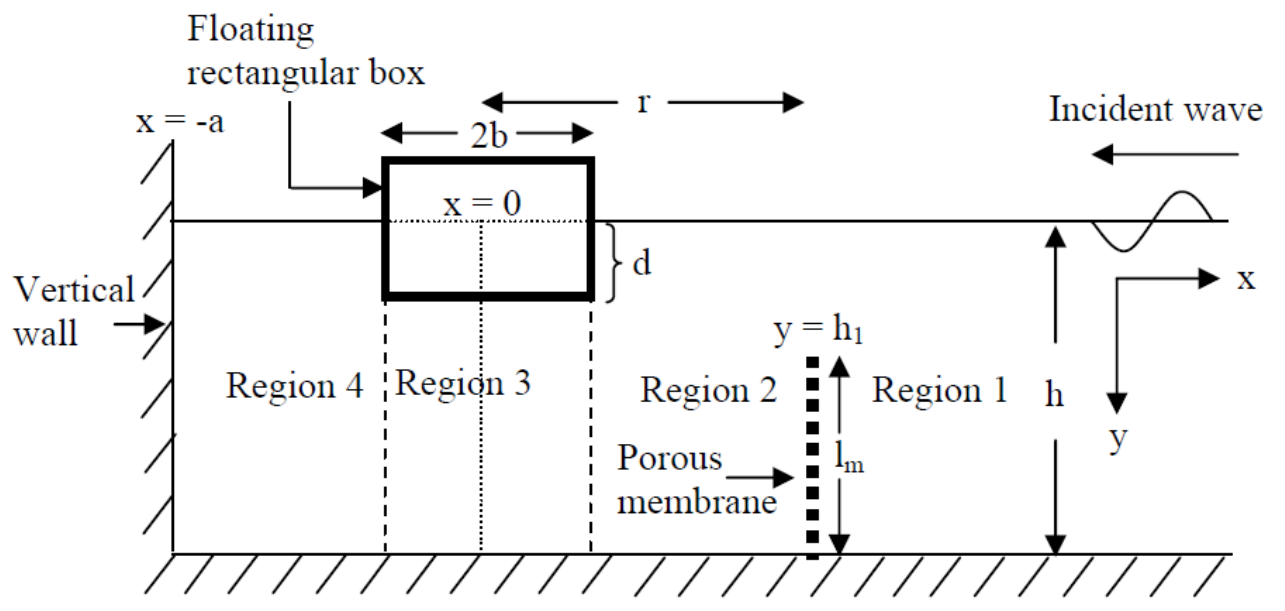

Figure 1. Schematic diagram.

Under the linear wave theory, the fluid is assumed to be inviscid, incompressible and the motion is irrotational and harmonic in time with angular frequency $\omega$. Hence, there exists a velocity potential of the form $\Phi(x, y, t)=\operatorname{Re}\left[\phi(x, y) e^{-i \omega t}\right]$, where $\phi(x, y)$ satisfies the Laplace equation in the fluid domain along with the standard free surface boundary condition. The normal velocity on the rigid bottom, the wall and on the wetted body boundary of the floating rectangular box vanishes.

The boundary condition on the porous membrane based on the Darcy's law is given by (see SAHOO et al. (2000), and YIP et al. (2002)):

$$
\partial \phi_{j} / \partial x=i k_{0} G\left(\phi_{2}-\phi_{1}\right)-i \omega \xi, x=r, h_{1}<y<h, j=1,2 \text {, }
$$

where $\mathrm{G}$ is the complex porous effect parameter and is given by (see SAHOO et al. (2000), and YU (1995)):

$$
G=G_{r}+i G_{i}=\gamma(f+i s) /\left\{k_{0} d_{m}\left(f^{2}+s^{2}\right)\right\}
$$


$\gamma$ is the porosity constant, which is defined as the ratio of the porous area to the whole area of the membrane, $f$ is the resistance force coefficient, $s$ is the inertial force coefficient, $d_{m}$ is the thickness of the membrane, $k_{0}$ is the incident wave number and $\xi(y)$ is the spatial horizontal deflection of the membrane. The dissipation of the incident wave energy is related to the resistant effect of the membrane, whereas the inertial force coefficient causes the phase shift of the wave motion. The membrane becomes impermeable when $|G|$ tends to zero, while the membrane becomes transparent for $|G|$ tending to infinity. It is assumed that $\xi(y)$ is small compared to the wavelength. The membrane deflection $\xi(y)$ is determined by solving the differential equation:

$d^{2} \xi / d y^{2}+m \omega^{2} \xi / T=-i \omega \rho\left(\phi_{2}-\phi_{1}\right) / T, x=r, h_{1}<y<h$,

where $m$ is the membrane mass per unit area and $T$ is the tension per unit length along the membrane length. The fixed end edge conditions are prescribed as:

$\xi\left(h_{1}\right)=\xi(h)=0$.

Assuming that the velocity and pressure are continuous across the gaps, the matching conditions are given by:

$$
\begin{array}{ll}
\partial \phi_{3} / \partial x=\partial \phi_{4} / \partial x, \phi_{3}=\phi_{4}, & x=-b, d<y<h, \\
\partial \phi_{2} / \partial x=\partial \phi_{3} / \partial x, \phi_{2}=\phi_{3}, & x=b, d<y<h, \\
\partial \phi_{1} / \partial x=\partial \phi_{2} / \partial x, \phi_{1}=\phi_{2}, & x=r, 0<y<h_{1} .
\end{array}
$$

The wave field satisfies the usual far field radiation condition.

\section{Method of solution}

The velocity potentials $\phi_{1}, \mathrm{i}=1, \ldots, 4$ in the four regions are expanded in terms of appropriate eigenfunctions as in BHATTACHARJEE \& GUEDES SOARES (2011), who analyzed the oblique wave interaction with a floating structure near a wall having a stepped bottom by the method of eigenfunction expansion. The same method is used here to study the effect of porous membrane barrier in water of uniform depth. Using the conditions in Eq. (1) and (5a, 5b, 5c) and applying the orthogonal properties of the eigenfunctions, a linear system of algebraic equations is obtained to determine the unknowns. The surface elevations in region 1, 2 and 4 are evaluated from the kinematic boundary condition and the membrane deflection is obtained from Eq. (3).

\section{Results and discussions}

In the present section, numerical results for the free surface wave elevations $\eta_{1}, \eta_{2}$ and $\eta_{4}$ in region 1,2 and 4 , respectively, and the membrane deflection $\xi$ are discussed for different values of the dimensionless membrane tension $T^{\prime}=T / \rho g h^{2}$ and porosity constant $G$. The following numerical values are kept fixed throughout the computations: $\rho=1025 \mathrm{~kg} \mathrm{~m}^{-3}, g=9.81 \mathrm{~m} \mathrm{~s}^{-2}, h=50 \mathrm{~m}, a=50 \mathrm{~m}, b=10 \mathrm{~m}, d=2 \mathrm{~m}$ and $m^{\prime}=m / \rho h=0.1$. It is observed that the infinite series sums converge for $N=15$ to 20 terms and hence is taken 
La connaissance de la Mer :

un vecteur du développement durable en Méditerranée

as $N=20$ for all the numerical computations. This shows that the method is efficient in terms of computation time and memory.

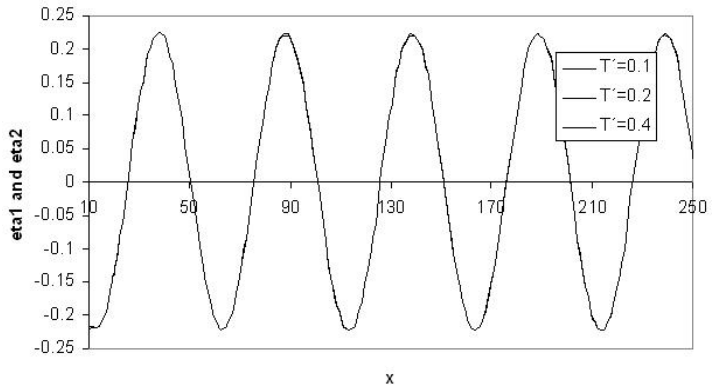

Figure 2. Free surface elevations $\eta_{1}$ and $\eta_{2}$ in regions 1 and 2 for $\omega \sqrt{ }(d / g)=0.5$, $G=1+2 i, r=20 \mathrm{~m}, h_{1}=10 \mathrm{~m}$ and dimensionless tension $T^{\prime}=0.1,0.2,0.4$.

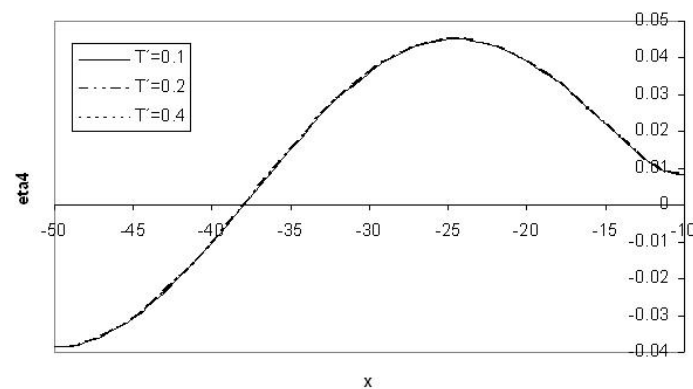

Figure 3. Free surface elevation $\eta_{4}$ in region 4 for $\omega \sqrt{ }(d / g)=0.5, G=1+2 i, r=20$ $m, h_{1}=10 m$ and dimensionless tension $T^{\prime}=0.1,0.2,0.4$.

Numerical computation shows that the reflection of incoming waves is always very high, which is obvious as the incoming waves get reflected by the rigid floating structure and the rigid wall. Figure 2 shows that the wave amplitude and the wavelength do not change significantly due to the presence of the membrane. This is perhaps due to the reason that the membrane is submerged below the free surface and the gravity waves are propagating on the free surface.

Figure 3 shows that the free surface elevation $\eta_{4}$ in region 4 is almost identical for different membrane tension. However, the wave amplitude is reduced significantly in comparison to the incident wave region. Hence, the wave load on the wall will be less than what it will experience if it is subject to direct incident wave attack.

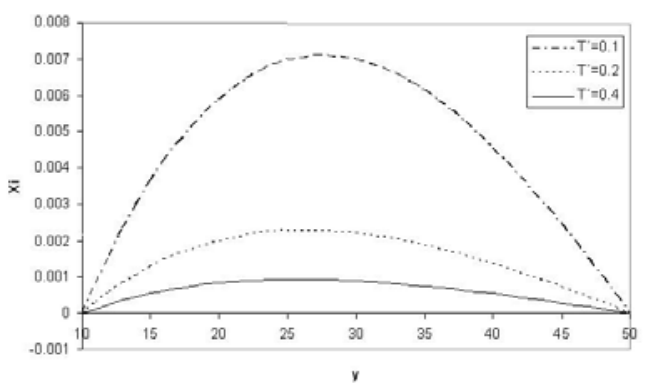

Figure 4. Membrane deflection $\xi$ for $\omega \sqrt{ }(d / g)=0.5, G=1+2 i, r=20 m, h_{1}=10 \mathrm{~m}$ and dimensionless tension $T^{\prime}=0.1,0.2,0.4$.

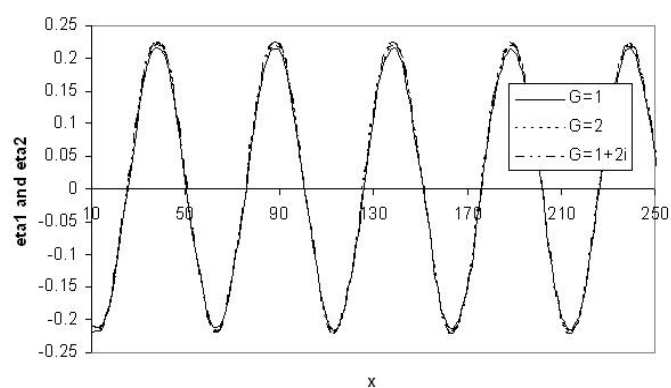

Figure 5. Free surface elevations $\eta_{1}$ and $\eta_{2}$ in regions 1 and 2 for $\omega \sqrt{ }(d / g)=0.5$, $T^{\prime}=0.4, r=20 \mathrm{~m}, h_{1}=10 \mathrm{~m}$ and porosity constant $G=1,2,1+2 i$.

Figure 4 shows that the membrane deflection $\xi$ reduces as $T^{\prime}$ along the membrane increases. Further, $\xi=0$ at the two ends, which supports the theoretical assumption of 
fixed edge conditions. Figures 5 and 6 show that the effect of $G$ on the propagation of surface waves in region 1,2 and 4 is almost negligible. It is noticed that the wave amplitude in region 4 increases for non zero real and imaginary parts of $G$. This is due to the fact that for $|G|>1$, the porous nature of the barrier increases and more waves pass through the barrier. The results assert that the effect of the submerged partial barrier is negligible as most of the incoming wave energy is concentrated near the free surface.

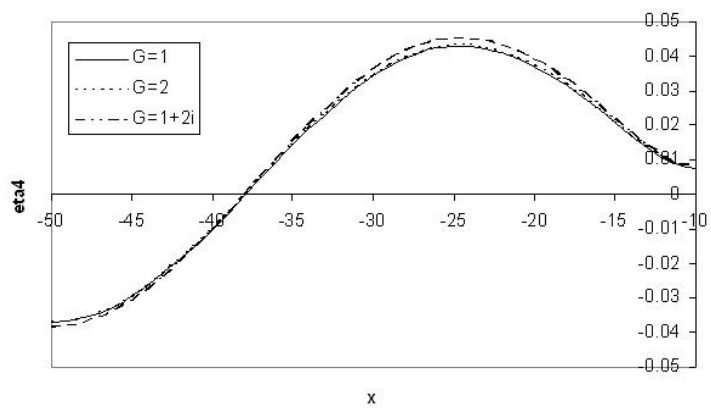

Figure 6. Free surface elevation $\eta_{4}$ in region 4 for $\omega \sqrt{ }(d / g)=0.5, T^{\prime}=0.4, r=20 \mathrm{~m}$, $h_{1}=10 \mathrm{~m}$ and porosity constant $G=1,2,1+2 i$.

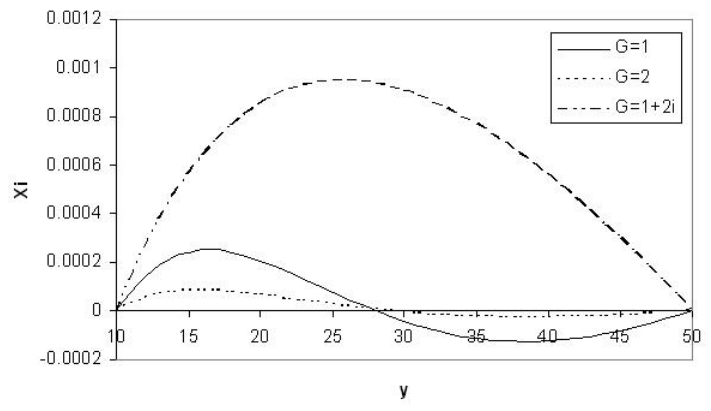

Figure 7. Membrane deflection $\xi$ for $\omega \sqrt{ }(d / g)=0.5, T^{\prime}=0.4, r=20 \mathrm{~m}, h_{1}=10 \mathrm{~m}$ and porosity constant $G=1,2,1+2 i$.

Figure 7 shows the variation of membrane deflection for various values of $G$. It shows that as the imaginary part of $G$ increases, the porous nature is reduced due to the dominance of the inertial effect. Therefore, dissipation of the incoming wave energy reduces and as a consequence the membrane deflection increases. On the other hand, for zero inertial effect, the membrane experiences sinusoidal deflections.

\section{Conclusions}

The performance of a partial bottom touching porous membrane as a breakwater to protect a floating structure near a wall is analyzed in the linear wave theory. The method of eigenfunction expansion is utilized to obtain an analytic solution. The method is efficient in terms of computer memory and time. It is observed that the effect of membrane tension and porosity constant on the free surface elevations in region 1 and 2 is almost negligible. However, the deflection of the membrane depends largely on these parameters. This is perhaps due to the reason that the partial barrier is submerged at the bottom and wave energy of the free surface gravity waves is concentrated near the free surface. The present results suggest more detailed study of different membrane configurations and comparison of results for a better understanding of the physics.

\section{Acknowledgements}

The first author has been funded by FCT, Fundação para a Ciência e a Tecnologia, Ministério da Ciência, Tecnologia e Ensino Superior under contract $\mathrm{n}^{\circ}$ 
La connaissance de la Mer :

un vecteur du développement durable en Méditerranée

SFRH/BPD/47210/2008. The work has been performed within the project "FLOATERS - Wave Interaction with Floating Structures in Shallow Water" financed by FCT, (Fundação para a Ciência e Tecnologia) under contract PTDC/ECM/ 111242/2009.

\section{References}

BHATTACHARJEE J., GUEDES SOARES C. (2011). Oblique wave interaction with a floating structure near a wall with stepped bottom, Ocean Engineering., 38, pp 15281544. doi:10.1016/j.oceaneng.2011.07.011

CHWANG A.T., CHAN A.T. (1998). Interaction between porous media and wave motion, Ann. Rev. Fluid Mech. 30, pp 53-84.

KEE S.T., KIM M.H. (1997). Flexible membrane wave barrier. II: floating/submerged buoy-membrane system. ASCE Journal of Waterway, Port, Coastal, and Ocean Engineering, 123, pp 82-90. doi:10.1061/(ASCE)0733-950X(1997)123:2(82)

KIM M.H., KEE S.T. (1996). Flexible-membrane wave barrier. I: analytic and numerical solutions. ASCE Journal of Waterway, Port, Coastal, and Ocean Engineering 122, pp 46-53. doi:10.1061/(ASCE)0733-950X(1996)122:1(46)

LEE M.M., CHWANG A.T. (2000). Scattering and radiation of water waves by permeable barriers. Physics of Fluids, 12, pp 54-65.

LEE W.K., LO E.Y.M. (2002). Surface-penetrating flexible membrane wave barrier of finite draft. Ocean Engineering, 29, pp 1781-1804. doi:10.1016/S0029-8018(02)00007-0

LO E.Y.M. (2000). Performance of a flexible membrane wave barrier of a finite vertical extent. Coastal Engineering Journal, 42, pp 237-251. doi:10.1142/\$0578563400000110 SAHOO T., LEE M.M., CHWANG, A.T. (2000). Trapping and generation of waves by vertical porous structures. J. Engng. Math., 126(10), pp 1074-1082.

SURESH KUMAR P., MANAM S.R., SAHOO T. (2007). Wave scattering by flexible porous vertical membrane barrier in a two-layer fluid. J. Fluids Structures, 23, pp 633647. doi:10.1016/j.jfluidstructs.2006.10.011

YIP T.L., SAHOO T., CHWANG A.T. (2002). Trapping of surface waves by porous and flexible structures. Wave Motion, 35, pp 41-54. doi:10.1016/S0165-2125(01)00074-9 YU X.P. (1995). Diffraction of water waves by porous breakwaters. J. Waterway, Port, Coastal, and Ocean Engineering, ASCE 121 (6), pp 275-282. doi:10.1061/(ASCE)0733950X(1995)121:6(275) 